\title{
IMPACT OF HOMOGENEITY INDEX ON RESPONSE RATE AND SURVIVAL IN HEAD AND NECK CANCER PATIENTS TREATED WITH IMRT
}

\author{
Flowerlit Thomas ${ }^{1}$, Suresh Kumar K², Mintu Mathew ${ }^{3}$, Kiran $P^{4}$
}

${ }_{1}^{1}$ Assistant Professor, Department of Radiotherapy, Cancer Care Center, Government Medical College, Kottayam, Kerala, India. ${ }^{2}$ Additional Professor, Department of Radiotherapy, Cancer Care Center, Government Medical College, Kottayam, Kerala, India. 3 Senior Resident, Department of Radiotherapy, Cancer Care Center, Government Medical College, Kottayam, Kerala, India. ${ }_{4}^{4}$ Senior Resident, Department of Radiotherapy, Cancer Care Center, Government Medical College, Kottayam, Kerala, India.

\section{ABSTRACT}

\section{BACKGROUND}

Homogeneity Index (HI) is a measurement used in conformal radiotherapy to evaluate the uniformity of dose distribution in the treatment volume. There are many controversies existing in the clinical usage of Homogeneity Index like the ideal formula to be used to calculate it, which type of radiotherapy (3DCRT/IMRT/SRS) can be evaluated using HI and what its clinical implication is. Even though the ultimate aim of radiation treatment is to improve local control and survival of the patient, there is scarcity of literature as to whether Homogeneity Index has any direct impact on patient survival

\section{MATERIALS AND METHODS}

This retrospective cohort study of 50 patients with head and neck tumours were treated with radical IMRT with concurrent chemotherapy were retrospectively analyzed. Homogeneity Index was calculated using 5 standard formulas and each was correlated with 3-month response rates and local recurrence free survival. Cox regression analysis and multivariate analysis were done to assess the survival and response rates respectively.

\section{RESULTS}

When analyzed together, none of the homogeneity indices showed any direct impact on survival or response rates in patients which were statistically significant. But subgroup analysis showed that when divided into sub sites, the homogeneity index calculated by (D5-D95)/Dp was statistically significant in predicting the local recurrence free survival. The impact of the value on survival when divided into individual sub sites were not analyzed as the sample size was too low.

\section{CONCLUSION}

HI calculated in this study by D5-D95/Dp seems to be the most statistically significant in predicting the local recurrence rates. But the real clinical impact of Homogeneity Index as a clinically important value still remains doubtful and it still does not warrant the status of a standalone value to evaluate plans.

\section{KEY WORDS}

IMRT, Homogeneity Index, Head and Neck, Survival.

HOW TO CITE THIS ARTICLE: Thomas F, Kumar SK, Mathew M, et al. Impact of homogeneity index on response rate and survival in head and neck cancer patients treated with IMRT. J. Evolution Med. Dent. Sci. 2019;8(01):62-66, DOI: 10.14260/jemds/2019/14

\section{BACKGROUND}

The treatment of Head and neck squamous cell carcinomas with radiotherapy presents a unique challenge in the field of Radiation Oncology. The shape of the target volumes, proximity to multiple critical organs and the dose response relationship all have led to the fact that head and neck carcinomas are one of the most common sites of application of advanced radiotherapy planning techniques. From conventional 2D techniques to proton therapy, the prompt application of newer technologies have seen to the improvements in survival rates and reduction of normal tissue toxicities in head and neck cancers.

'Financial or Other Competing Interest': None.

Submission 29-11-2018, Peer Review 25-12-2018,

Acceptance 31-12-2018, Published 07-01-2019.

Corresponding Author:

Dr. Suresh Kumar K,

Additional Professor,

Department of Radiotherapy,

Cancer Care Center, Government Medical College,

Kottayam, Kerala, India.

E-mail: drsureshkumar74@gmail.com

DOI: $10.14260 /$ jemds/2019/14
In India, head and neck cancers (Oral Cavity, Pharynx and Larynx) form the second most common malignancy in the population comprising of 14 percent of the total cancer burden. $^{1}$ The higher incidence of tobacco related malignancies and the advanced stage of presentation necessitate the use of radical concurrent chemo radiation as the treatment of choice for most laryngeal and pharyngeal cancers in our population. For minimization of toxicity while delivering tumouricidal doses, IMRT has emerged as the standard of care in head and neck cancers with some studies and a SEER database review suggesting survival advantage with IMRT. ${ }^{2}$

The physical goal of stereotactic radiosurgery (SRS) is to deliver a prescribed dose of radiation to the target geometry in as conformal a manner as possible, while spilling as little as possible into the surrounding normal tissue. The dosimetric indices like conformity index and homogeneity index were designed with the primary aim of comparing different plans for treating the same patient. They are extensively used in evaluation of highly conformal radiotherapy like in Cyber knife and Stereotactic Radiotherapy (SRT) but can also be also used to compare plans in other conformal treatments also. While Conformity indices (CIs) have been used to quantitatively evaluate the degree of dose conformity, 
Homogeneity Index (HI) has been described as an objective tool to analyse the uniformity of dose distribution in the target volume.

Conformity Index (CI) is commonly used to evaluate the acceptability of a plan. Conformity Index was initially described in ICRU 62.3 It is a complementary tool that attributes a score to a treatment plan or that can compare several treatment plans for the same patient. As the main aim of conformity Index is to compare plans for the same patient, in this Retrospective Descriptive study, conformity index has not been considered for comparison. But all plans included in the study had acceptable CI.

Radiation Therapy Oncology Group (RTOG) proposed guidelines for routine evaluation of stereotactic radiotherapy (SRT) plans based on several parameters, one of which was Homogeneity Index (HI). If the $\mathrm{HI}$ was $\leq 2$, treatment was considered to comply with the protocol, if between 2 to 2.5 , it was considered as minor violation, but if the index exceeded 2.5 , the violation of the protocol was considered to be major, but might nevertheless considered acceptable. ${ }^{4}$ Even though this index was developed initially to evaluate highly conformal plans, application of the $\mathrm{HI}$ in evaluation of Intensity modulated Radiotherapy (IMRT) ${ }^{5-7}$ and 3 dimensional conformal radiotherapy (3DCRT) ${ }^{8}$ has been done in literature with different results. The ideal formula to calculate homogeneity index is also still in question. Various definitions and formulae have been described in literature by various authors and organizations, none has been described as ideal or near ideal for calculating $\mathrm{HI}$.

Different formulae defined for calculation for homogeneity index are-

1. $\mathrm{HI}=\mathrm{D}_{5} / \mathrm{D}_{95}$; where $\mathrm{D}_{5}=$ minimum dose in $5 \%$ of the Planning Target Volume (PTV), indicating the "maximum dose", and $\mathrm{D}_{95}=$ minimum dose in $95 \%$ of the PTV, indicating the "minimum dose." 9

2. $\mathrm{HI}=\mathrm{D}_{2}-\mathrm{D}_{98} / \mathrm{D}_{\mathrm{p}} \times 100$; where $\mathrm{D}_{2}=$ minimum dose to $2 \%$ of the target volume indicating the "maximum dose", $\mathrm{D}_{98}=$ minimum dose to the $98 \%$ of the target volume, indicating the "minimum dose" and $\mathrm{D}_{\mathrm{p}}=$ prescribed dose. ${ }^{10}$

3. HI =Dmax/Dmin; where Dmax and Dmin represent the maximum and minimum point dose in the target volume respectively.

4. HI: $\mathrm{D}_{5}-\mathrm{D}_{95} / \mathrm{Dp} \times 100$; where $\mathrm{D} 5$ and $\mathrm{D} 95$ are the minimum dose in $5 \%$ and $95 \%$ of the target volume and $\mathrm{Dp}$ is the prescribed dose.

\section{Need for the Study}

There are various formulae described in different studies and none of them has been described as the standard for calculation of Homogeneity Index. Moreover, HI has always been described as a dosimetric parameter. It has always been used to compare treatment plans, different machines or different treatment techniques (e.g.: IMRT vs. Tomotherapy) etc., while assuming that a more homogenous plan will ensure better treatment delivery and in turn, assure better tumour control. But there is paucity of data relating this commonly used Index with tumour control and survival of the patient, which is the ultimate aim of treatment. This study tries to answer this question. We have analysed patient data to find out whether a better Homogeneity Index can in fact ensure better tumour control and translate this to survival benefit to the patient or should it continue to be used as a technical, dosimetric term only.

\section{MATERIALS AND METHODS}

This retrospective cohort study of 50 patients with inoperable loco regionally advanced squamous cell carcinoma of oropharynx, supraglottic larynx and hypopharynx treated with IMRT from 2103 to 2106 were retrospectively evaluated for the study. The parameters like age, name, Cancer Registry number, American Joint Cancer Committee (AJCC) $7^{\text {th }}$ edition stage, histology, radiation treatment schedule, radiation breaks if any, number of concurrent chemotherapy received and local control at 3 months after completion of treatment were retrieved from our tumour registry.

All patients with head and neck cancers were simulated in supine position after immobilizing with thermoplastic head and shoulders mask with five fixation points attached to a carbon fiber plate support. Computed Tomography (CT) axial slices of $3 \mathrm{~mm}$ thickness were acquired for each patient in treatment position. These images were transferred to Eclipse treatment planning system (TPS) Varian associates, Palo Alto, CA, USA workstation, and contouring is done. For each patient, on the CT images, slice per slice for each of the following structures were drawn by the oncologist.

Extent of Gross tumour volume primary (GTVP) is drawn based on clinical and CT findings. A margin of $1 \mathrm{~cm}$ is added to GTV to create clinical target volume of primary (CTVP). Another $5 \mathrm{~mm}$ margin is given for setup uncertainty to create planning target volume (PTVP). Nodal regions are delineated as per the DAHANCA (Danish Head and Neck Cancer Group), RTOG (Radiation Therapy Oncology group), and EORTC (European Organization for research) and Treatment of Cancer consensus guidelines for delineation of nodal regions 2013 update. PTV1 (planning target volume 1 which includes gross primary and nodal disease with proper margins) receives 66-70 Gy in 30-33 fractions while the PTV2 (Planning target volume 2 which includes uninvolved nodal levels) receives 50-54 Gy in 30-33 fractions.

The contouring of target volumes and the treatment plan of the subjects was extracted from the treatment planning system and reviewed.

The Dose Volume Histograms (DVH) of these plans were reviewed and the necessary data for calculation of Homogeneity index (HI) with the following formulae was extracted.

\section{Outcome Measurement}

\section{Data Management and Statistical Analysis}

Collected data was coded and entered in Microsoft Excel sheet and analysis was done using SPSS software. Histology, pathologic grade, maximum tumour diameter, maximum nodal diameter, stage, radiation breaks, number of days to complete radiation treatment and number of concurrent chemotherapies received were considered as confounding factors and were assessed using Cox regression model. The impact of HI on local control was assessed using Chi Square Test. $\mathrm{P}$ value less than 0.05 was considered as significant. A subgroup analysis for the 3 individual sub sites and their relation to $\mathrm{HI}$ was also be done. 


\section{Ethical Consideration}

The only ethical consideration in this study is regarding the confidentiality of the data collected and so individual consent from each patient has not been obtained. Patients have been informed on phone about the study and permission taken for data collection. Permission has been obtained from the Head of the Department of Radiotherapy to conduct this study.

\section{RESULTS}

50 patients were included in the study. The median age at presentation was 58 years. 13 patients were treated for carcinoma hypopharynx, 26 for carcinoma oropharynx and 11 patients were treated for carcinoma larynx.

\begin{tabular}{|c|c|c|}
\hline Site & & \\
\hline & Frequency & Percent \\
\hline Hypopharynx & 13 & 26 \\
\hline Oropharynx & 26 & 52 \\
\hline Larynx & 11 & 22 \\
\hline Total & $\mathbf{5 0}$ & $\mathbf{1 0 0}$ \\
\hline \multicolumn{2}{|c|}{ Table 1. Distribution of Patients by Subsite } \\
\hline
\end{tabular}

The mean tumour size was $3.68 \mathrm{~cm}$ and the mean size of the largest node was $1.6 \mathrm{~cm} .35$ patients $(70 \%)$ presented in stage IVA while the remaining was of stage 3 . The most common histological grade of squamous cell carcinoma was grade 2 , in $64 \%$ patients while $28 \%$ had grade 1 and $6 \%$ had grade 3 disease. 1 patient had non-squamous histology.

$36 \%$ patients had 3 cycles of neoadjuvant chemotherapy. The most common site in patients who received neoadjuvant chemotherapy was hypopharynx. $30 \%$ patients received 2 cycles and $30 \%$ received no neoadjuvant chemotherapy. Majority of them were given concurrent weekly cisplatin except 2 patients who didn't receive concurrent chemotherapy due to comorbidities. The median number of concurrent chemotherapy cycles received was 5 .

Among the IMRT schedules, 23 patients received 66 Gy/30 fractions while 26 received 70 Gy in 33 fractions. 1 patient received 60 Gy in 28 fractions.

Response rate at 3 months was analysed by RECIST criteria $1.1^{11} \quad(\mathrm{CR}=$ Complete Response, $\mathrm{PR}=$ Partial Response). All patients had at least partial response with objective response rate of $100 \%$.

\begin{tabular}{|c|c|c|}
\hline Response at 3 Months & & \\
\hline & Frequency & Percent \\
\hline CR & 43 & 86 \\
\hline PR & 7 & 14 \\
\hline Total & $\mathbf{5 0}$ & $\mathbf{1 0 0}$ \\
\hline \multicolumn{2}{|c|}{ Table 2. Response at 3 Months Post Treatment } \\
\hline
\end{tabular}

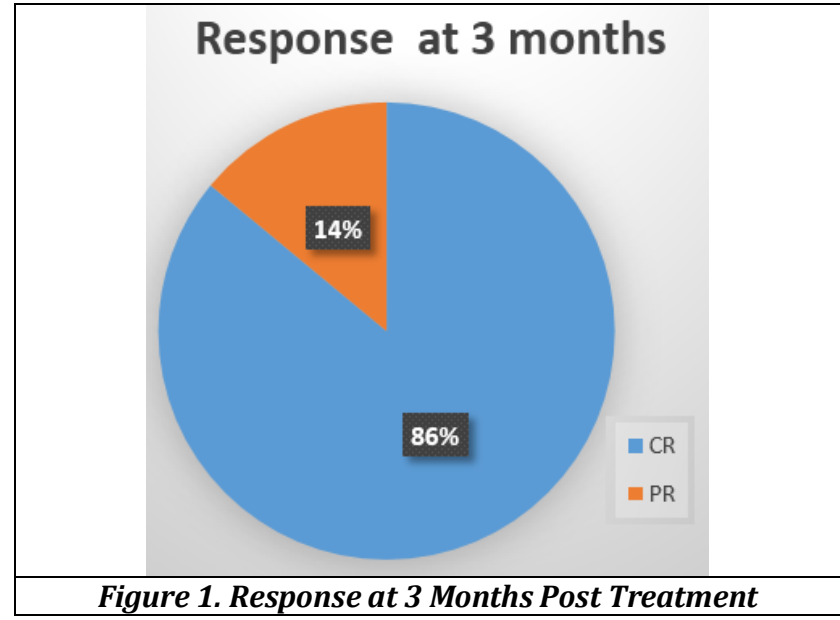

Till the end of study period, 16 patients were found to have recurrences 8 patients each had nodal and local failures.

\begin{tabular}{|c|c|c|}
\hline Recurrence & & \\
\hline & Frequency & Percent \\
\hline No & 34 & 68 \\
\hline Yes & 16 & 32 \\
\hline Total & $\mathbf{5 0}$ & $\mathbf{1 0 0}$ \\
\hline \multicolumn{2}{|c|}{ Table 3. Recurrence Rates }
\end{tabular}

In the 16 patients who had recurrence, 7 were having partial response and 9 had complete response. All 7 patients who had partial response at 3 months had recurrence.

Chi square tests revealed statistically significant correlation between response rates and recurrence $(p=0.000)$ but did not reveal any significant correlation of recurrence with sub site, stage, size of node/ primary or the schedule of RT used.

Kaplan Meir estimate revealed a mean survival of $68 \%$ at the end of study period.

Homogeneity indices were calculated by the formulae as mentioned above and was correlated with the recurrence free survival and response rates at 3 months.

Point-biserial correlation was used to analyse the correlation between responses at 3 months vs. the homogeneity indices. No statistically significant correlation was seen between any of the values of HI calculated by various formulae and the response rates. $\mathrm{P}$ value for all 4 indices $>0.05$

Cox regression was used to analyse the relation between recurrence free survival rates and the homogeneity indices. Here also, no statistically significant correlation was seen. $\mathrm{P}$ value for all 4 indices $>0.05$.

\begin{tabular}{|c|c|c|c|c|c|c|c|c|}
\hline & \multirow{2}{*}{ B } & \multirow{2}{*}{ SE } & \multirow{2}{*}{ Wald } & \multirow{2}{*}{ df } & \multirow{2}{*}{ Sig. } & \multirow{2}{*}{$\operatorname{Exp}(B)$} & \multicolumn{2}{|c|}{$95.0 \%$ CI for $\operatorname{Exp}(\mathrm{B})$} \\
\hline & & & & & & & Lower & Upper \\
\hline $\mathrm{HI}=\mathrm{Dmax} / \mathrm{Dmin}$ & -.986 & .652 & 2.286 & 1 & .131 & .373 & .104 & 1.339 \\
\hline $\mathrm{Hi}=\mathrm{D}_{5} / \mathrm{D}_{95}$ & -171.772 & 119.453 & 2.068 & 1 & .150 & .000 & .000 & $1.198 \mathrm{E} 27$ \\
\hline $\mathrm{HI}=\mathrm{D}_{2}-\mathrm{D}_{98} / \mathrm{D}_{\mathrm{p}} \times 100$ & -.206 & 5.096 & .002 & 1 & .968 & .814 & .000 & $1.771 \mathrm{E} 4$ \\
\hline HI: $D_{5}-D_{95} / \mathrm{Dp} \times 100$ & 218.831 & 145.897 & 2.250 & 1 & .134 & $1.089 \mathrm{E} 95$ & .000 & $1.678 \mathrm{E} 219$ \\
\hline
\end{tabular}

Table 4. Cox Regression Analysis between HI and Recurrence Free Survival

A planned subgroup analysis was done by dividing the patients into subgroups based on the subsite of the disease. The subsite of the disease was used as a covariate in cox regression analysis. The results show that there was a statistically significant correlation between the homogeneity indices calculated by D5-D95/ Dp and D5/D95 and the recurrence frees survival $(\mathrm{P}$ value $=0.046)$ 


\begin{tabular}{|c|c|c|c|c|c|c|}
\hline & B & SE & Wald & df & Sig. & Exp (B) \\
\hline $\begin{array}{c}\mathrm{HI}=\mathrm{D}_{2-} \\
\mathrm{D}_{98} / \mathrm{D}_{\mathrm{p}} \times 100\end{array}$ & -5.664 & 7.582 & .558 & 1 & .455 & .003 \\
\hline $\begin{array}{c}\mathrm{HI}: \mathrm{D}_{5-} \\
\mathrm{D}_{95} / \mathrm{Dp} \times 100\end{array}$ & 296.798 & 148.427 & 3.999 & 1 & .046 & $\begin{array}{c}7.905 \mathrm{E}+ \\
128\end{array}$ \\
\hline $\mathrm{HI}=\mathrm{D}_{5} / \mathrm{D}_{95}$ & -226.816 & 116.656 & 3.780 & 1 & .052 & .000 \\
\hline $\begin{array}{c}\mathrm{HI}=\mathrm{Dm}_{\max } / \\
\text { Dmin }\end{array}$ & -1.470 & 1.120 & 1.723 & 1 & .189 & .230 \\
\hline
\end{tabular}

Table 6. Cox Regression Analysis between HI and Survival Divided into Subsites

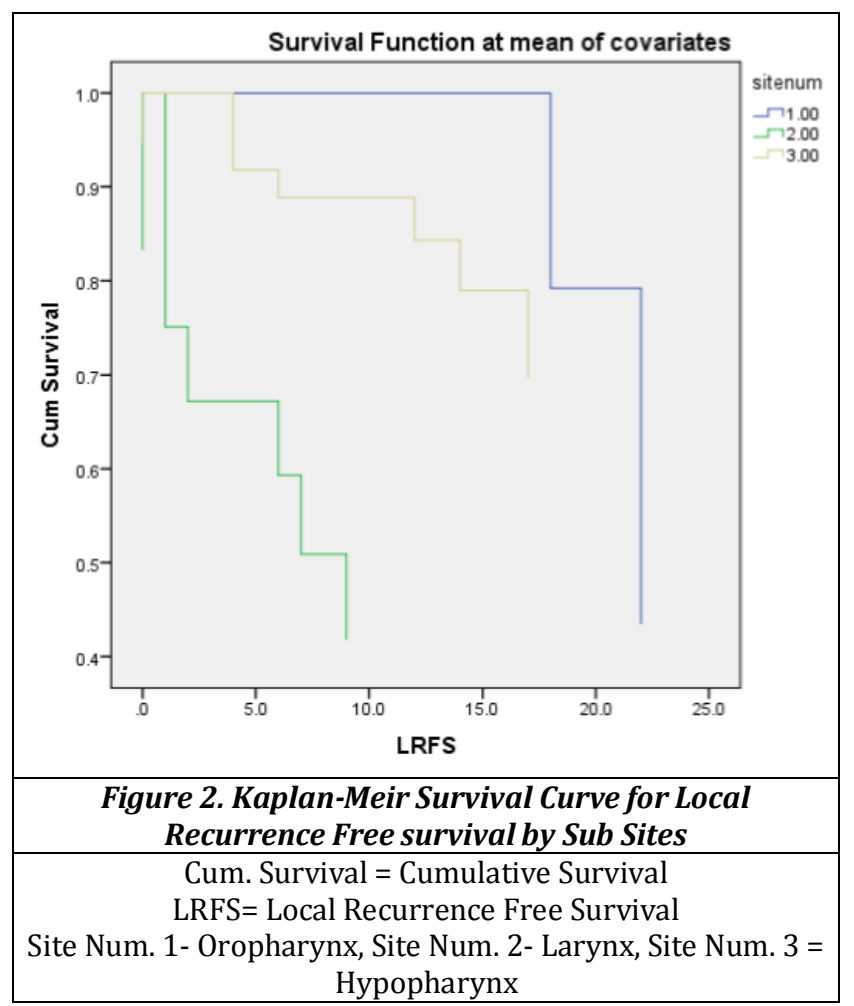

The significance of individual sub sites on survival rates could not be calculated as the study was not powered to do so.

\section{DISCUSSION}

The improvements in radiation therapy planning and delivery have led to the increasing use of Homogeneity Index as a measure of the efficiency of a radiation plan and is often used as the last decision-making tool when comparing two plans which meet all the other requirements of the oncologist and the physicist/dosimetrist. The clinical implication of $\mathrm{HI}$ has never been validated and overdependence on such a nonvalidated tool to make decisions which have direct clinical implication is not advisable. This study attempts to validate the direct impact of HI on recurrence free survival in head and neck cancer patients treated with IMRT. IMRT and not stereotactic radiation therapy (SRT) was chosen for this study because IMRT is more commonly used in radical treatment of patients than SRT especially in head and neck cancer patients.

Survival estimates for patients treated with SRT would not lead to meaningful results as it is mostly indicated in metastatic lesions.

The baseline characteristics of patients were in accordance to the institutional and regional averages. Majority of patients were having carcinoma oropharynx, and most were tobacco related. Some patients received neoadjuvant chemotherapy (Mostly in hypopharyngeal cancers) and almost all received concurrent chemotherapy.

At 3 months post RT, all patients had at least partial response. Response rates at 3 months were significantly correlated with relapse free survival with partial responders having worse survival than complete responders. All 7 patients who had partial response at 3 months had recurrence by the end of study period. But no significant correlation was seen with sub site, stage, size of node/ primary or the schedule of RT used. By the results of this study, it is seen that complete response to RT is the most important factor influencing survival in head and neck cancer patients regardless of site, size and stage.

Homogeneity Indices were calculated using various formulae which are commonly used in in clinical practice. These formulae have been validated dosimetrically 10,12 but clinical validation has not been attempted, probably because these formulae were developed for dosimetric comparison only. The study by Kataria et al ${ }^{12}$ states that increasing the prescribed dose improves the homogeneity index which may translate into a better clinical outcome by dose escalation but a direct correlation has never been established .

No statistically significant correlation was seen between response rates or recurrence rates with $\mathrm{HI}$ calculated by any formulae in the study. This implies that when taking head and neck malignancies as a whole, homogeneity index is not a clinically valid surrogate for improved patient survival. Thus, while it may be used to compare different plans for the same patient, it cannot be used as a predictive factor.

The validation and justification of many newer treatment modalities is done dosimetrically by comparing the homogeneity indices alone. ${ }^{13}$ The results of this study also points at the dangers of such comparisons .But as each patient's disease is a myriad of different factors which influence the results of treatment and because no patient can be treated with two different plans to compare outcomes, we are left with such indices only to select a better treatment plan. But still it must be remembered that a plan with a better homogeneity index is not necessarily a better plan.

Cox regression was used with the sub sites as subgroups to perform subgroup analysis. The results showed a statistically significant correlation with D5-D95 Homogeneity Index. This finding may be interpreted to indicate that in some sub sites, this homogeneity Index may be significantly correlated with survival. But further conclusions cannot be drawn from this finding. This study was not designed to evaluate the impact of homogeneity indices on individual sub sites or the impact of different homogeneity indices on each sub site. Various other confounding factors like difference in size of lesion by sub sites and difference in proximity to organs at risk across sub sites may also influence this finding. Further studies powered to estimate the impact of these indices across different sub sites will have to be undertaken to further determine the implications of this finding and to identify the specific sub site and the specific Homogeneity index valid in the sub site.

\section{Limitations of the Study}

The study was conducted retrospectively with only 50 patients and varying sub sites of primary. As the results of this study indicate that the correlation between recurrence and homogeneity index might be valid in selected sub sites, 
larger studies which are sub site specific studies would be advisable to clarify this issue.

\section{CONCLUSION}

There is no direct impact of Homogeneity Index calculated by any of the commonly used formulae on response to treatment or recurrence rates. This study rules out the utility of Homogeneity Index as a predictive factor for response or recurrence rate. The use of $\mathrm{HI}$ as a dosimetric tool needs further evaluation in larger trials.

\section{REFERENCES}

[1] GLOBOCAN 2012 (IARC) Section of Cancer. http://globocan.iarc.fr/Pages/fact_sheets_population. aspx.

[2] Beadle BM, Liao KP, Elting LS, et al. Improved survival using intensity-modulated radiation therapy in head and neck cancers: a SEER-Medicare analysis. Cancer 2014;120(5):702-10.

[3] Landberg T, Chavaudra J, Dobbs J, et al. Report 62. Journal of the International Commission on Radiation Units and Measurements 1999;os32(1):NP-NP.

[4] Shaw E, Kline R, Gillin M, et al. Radiation therapy oncology group: radiosurgery quality assurance guidelines. Int J Radiat Oncol Biol Phys 1993;27(5):1231-9.

[5] Beckham WA, Popescu CC, Patenaude VV, et al. Is multibeam IMRT better than standard treatment for patients with left-sided breast cancer? Int J Radiat Oncol Biol Phys 2007;69(3):918-24.

[6] Pathak P, Vashisht S. A quantitative analysis of intensity-modulated radiation therapy plans and comparison of homogeneity indices for the treatment of gynecological cancers. J Med Phys 2013;38(2):6773.
[7] Krishnan J, Shetty J, Rao S, et al. Comparison of rapid arc and intensity-modulated radiotherapy plans using unified dosimetry index and the impact of conformity index on unified dosimetry index evaluation. J Med Phys 2017;42(1):14-7.

[8] Salimi M, Abi KST, Nedaie HA, et al. Assessment and comparison of homogeneity and conformity indexes in step-and-shoot and compensator-based intensity modulated radiation therapy (IMRT) and threedimensional conformal radiation therapy (3D CRT) in prostate cancer. J Med Signals Sens 2017;7(2):102-7.

[9] Gong Y, Wang J, Bai S, et al. Conventionallyfractionated image-guided intensity modulated radiotherapy (IG-IMRT): a safe and effective treatment for cancer spinal metastasis. Radiat Oncol 2008;3:11.

[10] Wu Q, Mohan R, Morris M, et al. Simultaneous integrated boost intensity-modulated radiotherapy for locally advanced head-and-neck squamous cell carcinomas. I: dosimetric results. Int J Radiat Oncol Biol Phys 2003;56(2):573-85.

[11] Eisenhauer EA, Therasse P, Bogaerts J, et al. New response evaluation criteria in solid tumours: revised RECIST guideline (version 1.1). Eur J Cancer 2009;45(2):228-47.

[12] Kataria T, Sharma K, Subramani V, et al. Homogeneity index: an objective tool for assessment of conformal radiation treatments. J Med Phys 2012;37(4):207-13.

[13] Ceylan C, Kucuk N, Bas Ayata H, et al. Dosimetric and physical comparison of IMRT and CyberKnife plans in the treatment of localized prostate cancer. Rep Pract Oncol Radiother 2010;15(6):181-9. 